\title{
A Randomized Crossover Evaluation of Antianginal Efficacy and Safety of Nitrolingual-Spray and Nitroglycerin Tablet Form in Coronary Artery Disease Patients
}

\author{
Kuo-Liong Chien ${ }^{a}$ Fung-Chang Sung ${ }^{b}$ Chia-Lun Chao ${ }^{a} \mathrm{Ta}^{-C h e n} \mathrm{Su}^{\mathrm{a}}$ \\ Ming-Fong Chen ${ }^{a}$ Yuan-Teh Lee ${ }^{a}$ \\ a Department of Internal Medicine, National Taiwan University Hospital, and 'bschool of Public Health, \\ National Taiwan University, Taipei, Taiwan, ROC
}

\section{Key Words}

Coronary artery disease $\cdot$ Treadmill exercise test .

Nitroglycerin $\cdot$ Clinical trial

\begin{abstract}
Twenty-eight coronary artery disease patients with more than $50 \%$ stenosis in at least one major coronary artery completed this randomized crossover clinical trial for the comparison of efficacy and safety of Nitrolingual-Spray and nitroglycerin (NTG) tablets. Exercise time was lengthened to $399.1 \mathrm{~s}$ (spray) or $408.5 \mathrm{~s}$ (tablets), compared to a baseline of $387.3 \mathrm{~s}$. Ischemic burden decreased to about $-4.0 \mathrm{~mm}$ with both forms, compared to $-7.5 \mathrm{~mm}$ at baseline (ANOVA: $\mathrm{p}=0.003$ ). The ischemic time improved to $137.2 \mathrm{~s}$ (spray) or $152.9 \mathrm{~s}$ (tablets), compared to $253.4 \mathrm{~s}$ at baseline (ANOVA: $p=0.005$ ). Patients taking tablets experienced more episodes of hypotension and/or headache compared to patients taking the spray. Nitrolingual-Spray is as effective and safe as NTG tablets for the treatment of symptomatic coronary heart disease.
\end{abstract}

Copyright @ 2000 S. Karger AG, Basel

\section{Introduction}

Sublingual nitroglycerin (NTG) is an established therapy for the treatment of effort angina [1]. Nonetheless, the pharmacological activity of NTG tablets diminishes gradually, particularly if it is not properly stored [2-6]. Tallett [7] suggested that patients have NTG tablets dispensed at least every 12 weeks, even when following instructions for optimal storage. Lack of knowledge about the tablet form limits the usefulness of the drug [8,9]. New formulations of NTG with greater stability are now available.

Several clinical trials have demonstrated that NTG spray has a more rapid action of angina relief than NTG tablets [10-13] due to its rapid absorption [14]. We conducted a randomized crossover clinical trial in Taiwan to compare the antianginal efficacy and safety of the most commonly used NTG tablets $(0.6 \mathrm{mg})$ with a new spray formulation (Nitrolingual-Spray, $0.4 \mathrm{mg}$ per puff; Pohl Boskamp, Hohenlockstedt, Germany) for treatment of coronary artery disease (CAD) patients with myocardial ischemia, documented by a treadmill exercise test (TxT).

Dr. Kuo-Liong Chien, Department of Internal Medicine National Taiwan University Hospital

No. 7 Chung-San Road, Taipei, Taiwan (ROC)

Tel. +8862 2397 0800, ext 7777, Fax +8862 23959911

E-Mail klchien@ha.mc.ntu.edu.tw 


\section{Patients and Methods}

Thirty CAD patients with $\geqslant 50 \%$ stenosis in at least one of the three major coronary arteries were recruited into this randomized crossover clinical trial. All patients gave informed consent. The hospital ethics review committee had approved the study. There were 25 men and 5 women (average age, $59.4 \pm 8.5$ years; table 1 ). All patients had symptomatic angina, documented CAD by coronary angiography, and a positive TxT. Patients with any of the following criteria were excluded: myocardial infarction within the previous 6 months, congestive heart failure (NYHA $\geqslant 3$ ), symptomatic valvular heart disease, or cardiomyopathy.

All patients performed a TxT without NTG tablets or spray to establish baseline performance. They were then randomly assigned to either the spray-to-tablet or tablet-to-spray groups (fig. 1). In the spray-to-tablet group, patients underwent a TxT immediately after

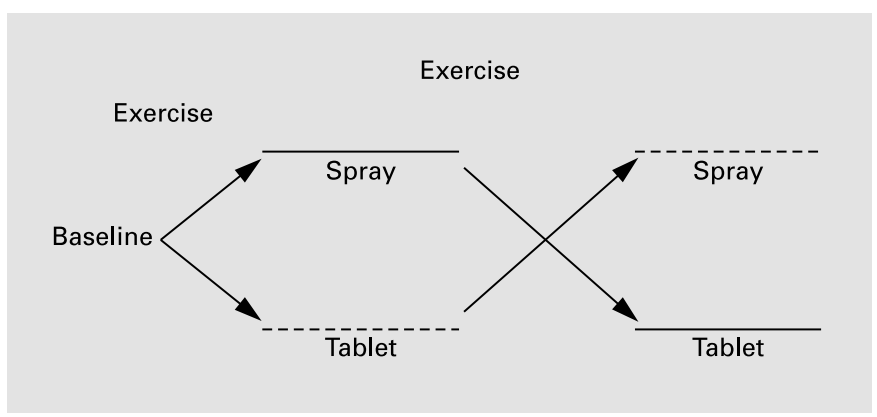

Fig. 1. Study protocol. — ST group: 1 puff $(0.4 \mathrm{mg})$ of NTG oral spray $\rightarrow 1$ tablet $(0.6 \mathrm{mg})$ of sublingual NTG. --- TS group: 1 tablet $(0.6 \mathrm{mg})$ of sublingual NTG $\rightarrow 1$ puff $(0.4 \mathrm{mg})$ of NTG oral spray. taking one 0.4-mg NTG spray puff. After resting for at least $30 \mathrm{~min}$, the patients took an NGT $0.6-\mathrm{mg}$ tablet sublingually and then performed another TxT. In the tablet-to-spray group, the drug form application was reversed. All TxT were performed using the standard Bruce protocol. ST-segment depression of at least $1.0 \mathrm{~mm}$ was considered significant for exercise-induced ischemia. The stress test was considered negative if at least $85 \%$ of the predicted maximal heart rate (defined as 220 - age in years) was achieved without significant ST-segment changes. Those patients with maximal heart rates $<85 \%$ of the predicted rate were excluded from the study. The end point of the TxT was extreme fatigue and exhaustion.

We compared the following variables of the two groups to baseline exercise performance: (1) exercise duration: the time from start to maximal exercise; (2) ischemic burden: total ST-segment depression during exercise, and (3) ischemic time: time duration from apperance to disappearance of ST-segment depression during exercise and resting periods. Secondary end points compared to baseline included rate-pressure product (RPP), blood pressure patterns, and adverse effects such as hypotension, palpitation, headache, and dizziness.

Efficacy was assessed in terms of exercise capacity by symptomlimited exercise time, total work performed, time to onset of angina, and ST-segment change.

\section{Statistical Analysis}

All values are presented as means $\pm \mathrm{SD}$. The results were analyzed for exercise period 1 and then for period 2, as a crossover design. Data were grouped by drug form and then analyzed. Analysis of variance was used to test the differences of measurements among baseline, and NTG spray and tablet treatments. The differences in test variables between NTG spray and tablets were analyzed using linear regression, with random effect incorporated in the model. Signifcance of differences in adverse events was tested using the $\chi^{2}$ or Fisher's exact test. $\mathrm{p}$ values $<0.05$ were considered significant for all parameters tested.
Table 1. Demographic characteristics of the study subjects

\begin{tabular}{lcc}
\hline & ST group $(\mathrm{n}=15)$ & TS group $(\mathrm{n}=15)$ \\
\hline Men & 12 & 13 \\
Women & 3 & 2 \\
Age, years (mean \pm SD) & $59.2 \pm 19.8$ & $59.6 \pm 5.6$ \\
$\quad$ Range & $40-75$ & $47-70$ \\
Body weight, kg (mean \pm SD) & $69.07 \pm 12.56$ & $69.06 \pm 6.42$ \\
Body height, cm (mean \pm SD) & $165.6 \pm 8.1$ & $168.8 \pm 5.8$ \\
History of angina & 10 & 10 \\
Hypertension & 7 & 8 \\
Diabetes mellitus & 4 & 1 \\
Hyperlipidemia & 13 & 9 \\
Smoking & & \\
$\quad$ Yes & 3 & 3 \\
$\quad$ No & 6 & 5 \\
$\quad$ Quit & 6 & 5 \\
\hline
\end{tabular}

ST $=1$ puff $(0.4 \mathrm{mg})$ of NTG oral spray $\rightarrow 1$ tablet $(0.6 \mathrm{mg})$ of sublingual NTG; TS $=$ 1 tablet $(0.6 \mathrm{mg})$ of sublingual NTG $\rightarrow 1$ puff $(0.4 \mathrm{mg})$ of NTG oral spray. 


\section{Results}

Of the 30 patients enrolled, 28 completed the protocol, and 2 patients withdrew because of severe headache while taking NTG tablets. The two treatment groups were similar in basic demographic characteristics (table 1). The mean heart rate, systolic blood pressure, diastolic blood pressure and RPP were similar among baseline, NTG spray, and NTG tablet treatment (table 2).

The duration of exercise increased from $387.3 \mathrm{~s}$ at baseline to $399.1 \mathrm{~s}$ with NTG spray treatment and $408.5 \mathrm{~s}$ with NTG tablet treatment ( $\mathrm{p}=0.26$; table 2$)$. The average ischemic burden was reduced from $-7.50 \mathrm{~mm}$ at baseline to $-3.97 \mathrm{~mm}$ with NTG tablets and $-4.04 \mathrm{~mm}$ with NTG spray (ANOVA: $p=0.003$ ). While the two treatment groups showed similar reductions in ST-segment depression, ischemic burden in both treatment groups was significantly less at baseline (fig. $2 \mathrm{a}$ ). The average ischemic time was also shortened with both forms of NTG compared with baseline (ANOVA: $p=0.005$, table 2, fig. 2b).
Two patients in the NTG spray group did not develop ST depression of $>1 \mathrm{~mm}$, even at the end of exercise. Thus, we used the heart rate and blood pressure values at the peak of exercise as indicators of ischemia development. The time required before ischemia developed during exercise was prolonged in both treatment groups (355.4 s with NTG spray and $336.9 \mathrm{~s}$ with NTG tablets) compared to baseline (287.6 s, $\mathrm{p}=0.30$, table 2$)$. Average heart rate, systolic blood pressure and RPP during ischemia were significantly higher with NTG spray than with NTG tablets. Figure 2c shows that greater stresses were required to develop ischemia in the spray group than in the tablet group.

There were no significant differences in the frequency and types of reported adverse events between the two treatment groups; nonetheless, more patients experienced hypotension, headache, and dizziness with NTG tablets ( 5 patients) than with NTG spray (0 patients; table 3$)$.

Table 2. Comparison of hemodynamic parameters in patients at baseline, with NTG spray, and NTG tablet groups

\begin{tabular}{|c|c|c|c|c|c|}
\hline & $\begin{array}{l}\text { Baseline } \\
(\mathrm{n}=28)\end{array}$ & $\begin{array}{l}\text { Spray } \\
(\mathrm{n}=28)\end{array}$ & $\begin{array}{l}\text { Tablet } \\
(\mathrm{n}=28)\end{array}$ & $\begin{array}{l}\text { Difference } \\
\text { spray vs. tablet }\end{array}$ & $\mathrm{p}$ value \\
\hline \multicolumn{6}{|l|}{ Initial } \\
\hline HR & $77.8 \pm 13.1$ & $76.1 \pm 12.1$ & $78.9 \pm 12.9$ & -2.7 & 0.18 \\
\hline SBP & $135.5 \pm 21.5$ & $134.5 \pm 12.3$ & $131.0 \pm 14.4$ & 3.5 & 0.14 \\
\hline DBP & $80.7 \pm 11.6$ & $82.4 \pm 10.8$ & $78.6 \pm 7.3$ & 3.7 & 0.11 \\
\hline RPP & $10,528.4 \pm 2,303.4$ & $10,233.7 \pm 1,852.0$ & $10,397.6 \pm 2,475$ & -156.2 & 0.68 \\
\hline \multicolumn{6}{|l|}{ Peak } \\
\hline HR & $143.3 \pm 16.9$ & $143.5 \pm 17.8$ & $144.0 \pm 18.2$ & -0.6 & 0.70 \\
\hline SBP & $172.1 \pm 29.4$ & $171.0 \pm 27.6$ & $170.3 \pm 24.3$ & 0.06 & 0.99 \\
\hline DBP & $80.7 \pm 14.4$ & $80.2 \pm 11.3$ & $80.9 \pm 11.8$ & -0.7 & 0.75 \\
\hline RPP & $24,676.7 \pm 4,941.4$ & $24,580.3 \pm 5.317 .3$ & $24,529.4 \pm 4,829.3$ & -48.5 & 0.95 \\
\hline Duration of exercise, $\mathrm{s}$ & $387.3 \pm 102.3$ & $299.1 \pm 99.8$ & $408.5 \pm 91.1$ & -10.2 & 0.26 \\
\hline Ischemic burden, $\mathrm{mm}^{*}$ & $-7.5 \pm 5.1$ & $-4.04 \pm 3.8$ & $-3.97 \pm 3.5$ & -0.07 & 0.86 \\
\hline Ischemic times, $* *$ & $253.4 \pm 183.7$ & $137.2 \pm 116.3$ & $152.9 \pm 101.7$ & -15.7 & 0.34 \\
\hline \multicolumn{6}{|c|}{ Conditions of ischemic development } \\
\hline Time to ischemia, $\mathrm{s}$ & $287.6 \pm 114.6$ & $355.4 \pm 111.2$ & $336.9 \pm 152.1$ & 18.5 & 0.30 \\
\hline HR & $127.1 \pm 15.9$ & $131.7 \pm 19.5$ & $125.2 \pm 19.17$ & 6.42 & 0.009 \\
\hline SBP & $163.9 \pm 20.7$ & $171.5 \pm 25.3$ & $156.9 \pm 24.5$ & 14.64 & 0.005 \\
\hline DBP & $80.8 \pm 12.1$ & $83.3 \pm 19.8$ & $77.4 \pm 10.2$ & 5.89 & 0.15 \\
\hline $\mathrm{RPP} * * *$ & $20,915.4 \pm 4,127.5$ & $22,741.0 \pm 5.563 .5$ & $19.826 .8 \pm 5.251 .1$ & $2,914.2$ & 0.002 \\
\hline
\end{tabular}

Crossover analyses were also performed to compare the means of various variables between spray and tablet groups, with $\mathrm{p}$ values.

$\mathrm{HR}=$ Heart rate; $\mathrm{SBP}=$ systolic blood pressure; $\mathrm{DBP}=$ diastolic blood pressure; ischemic burden = total ST-segment depression; ischemic time $=$ time from ST depression appearance to disappearance. ANOVA $* p=0.0028$, indicating total ST depression among the three treatments were significantly different. $* * p=0.0054, * * * p=0.046$. 
Fig. 2. Comparisons of ischemic burden (a), ischemic time (b), and RPP (c) at the onset of ischemia.
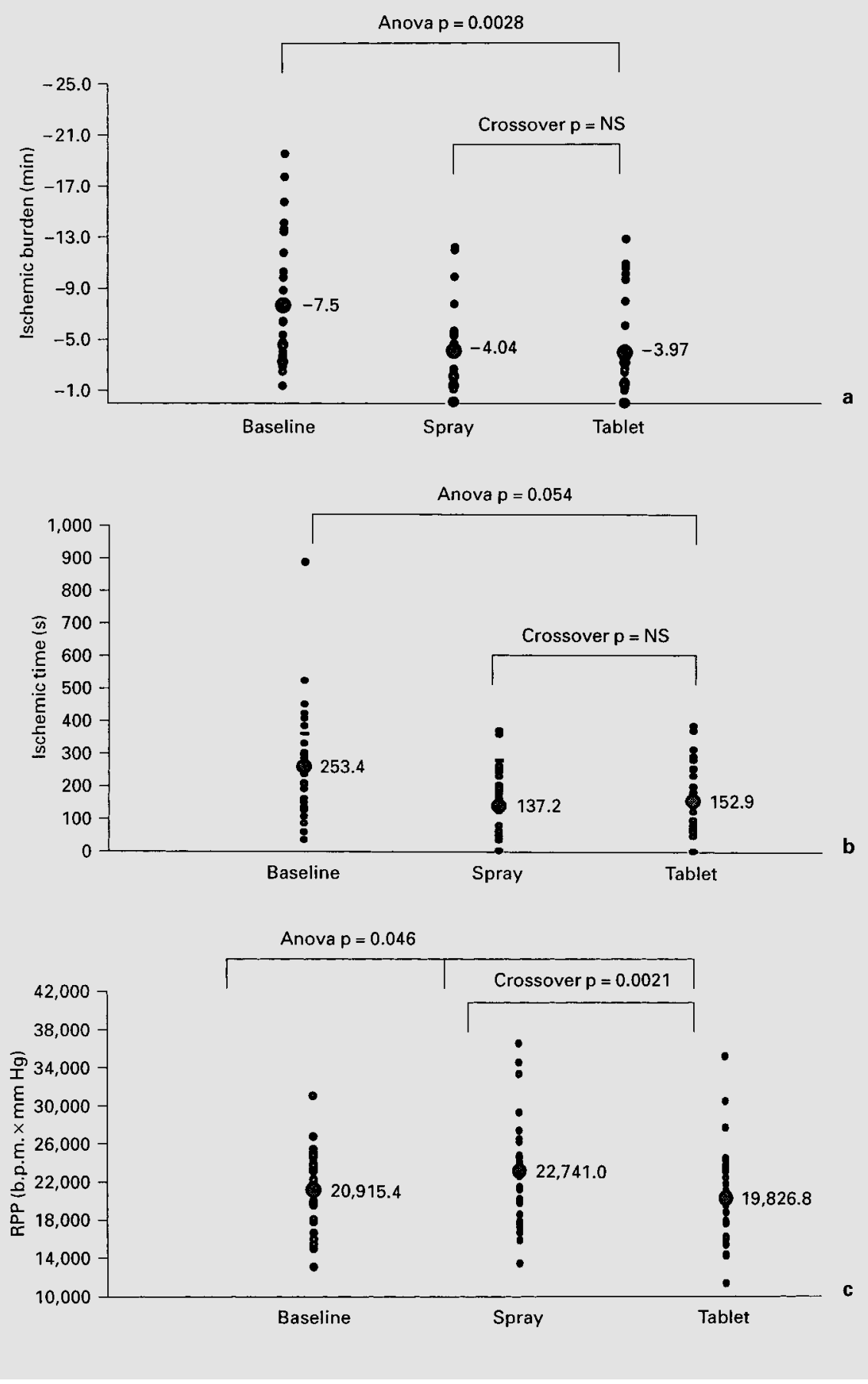

\section{Discussion}

This crossover clinical trial compared the effectiveness of NTG spray and tablets for treatment of exerciseinduced ischemia in CAD patients. The results demon- strated that exercise duration, total ST-segment depression, and ischemic time were improved with both spray and tablet forms compared with baseline. There were significant differences in heart rate, blood pressure, and RPP during exercise-induced ischemia between the two treat- 
Table 3. Adverse events with NTG spray and tablet treatments

\begin{tabular}{lll}
\hline Adverse events & $\begin{array}{l}\text { NTG spray } \\
(\mathrm{n}=30)\end{array}$ & $\begin{array}{l}\text { NTG tablets } \\
(\mathrm{n}=30)\end{array}$ \\
\hline Hypotension* & 0 & 5 \\
Palpitations & 0 & 3 \\
Headache & 1 & 5 \\
Dizziness & 1 & 5 \\
Edema & 0 & 0 \\
Nausea & 0 & 0 \\
Fatigue & 0 & 1 \\
\hline
\end{tabular}

Two patients withdrew due to severe headache with the tablet form. ${ }^{*} \mathrm{p}=0.051$ (Fisher's exact test).

ments. Patients taking the spray form seemed to tolerate higher stress before ischemia developed than patients taking the tablet form.

Previous clinical trials have reported several differences in efficacy between the two NTG spray and tablet formulations [10, 12]. NTG spray has a longer shelf life with no stability problems, but NTG tablets carried by patients are often out of date and have decreased efficacy $[5,9]$. While both tablet and spray NTG are convenient to use, the spray form may have a greater advantage, as it causes fewer headache and hypotension episodes than the tablet form. Although this was an actively controlled study, it was not double-blinded and, therefore, there may have been some information bias on the part of the observers or patients. However, the similar distributions of demographic and risk factors in both groups implies that the comparison is acceptable. In comparing NTG tablets $(0.6 \mathrm{mg})$ with the metered-dose NTG spray $(0.4 \mathrm{mg})$, the difference in formulation might account for the improved results in the NTG spray group in our study. Lee et al. [14] reported an earlier onset of action with NTG spray compared to tablets. We did not find significant differences between spray and tablet forms, except when ischemia developed.

Crossover designs reduce between-individual variance and improve the efficiency of the sample size [15]. On the other hand, crossover studies may suffer from carry-over effect and/or period effect. Because the elimination halflife following both sublingual administration of NTG spray and tablets ranges from 2.5 to $4.4 \mathrm{~min}$ [14], a carryover effect was avoided by taking a long enough rest before undergoing the next crossover treatment. The period effect was adjusted by a model fitting technique, incorporating random effect into the fitted linear regression model.

Based on the efficacy, adverse effects, storage convenience, and hemodynamic parameters, NitrolingualSpray should be considered as a therapy for CAD patients with angina pectoris. It may be a superior preparation compared to the tablet form and may have clinical advantages.

\section{Acknowledgment}

This study was supported by the Chang-Long Company, Taiwan, and the authors thank Miss Shu-Hui Chu and Miao-Chen Wang for the preparation of the manuscript.

\section{References}

1 Opie LH: Drugs and the heart: Nitrates. Lancet 1980;i:750-753.

2 Page DP, Carson NA, Buhr CA, Flinn PE, Wells CE, Randall MT: Stability study of nitroglycerin sublingual tablets. J Pharm Sci 1975; 64:140-147.

3 Fusari SA: Nitroglycerin sublingual tablets. 1. Stability of conventional tablets. J Pharm Sci 1973;62:122-129.

4 Russell VA, Lynch M: Storage of glyceryl trinitrate tablets in dispensing containers. Pharm $\mathbf{J}$ 1973;211:466-468

5 O'Hanrahan M, McGarry K, Kelly JG, Horgan J, O’Malley K: Diminished activity of glyceryl trinitrate. Br Med J 1982;284:1183-1184.

6 Marty J, Shaw J, Hunt D: The stability of glyceryl trinitrate tablets during patient use. Aust NZ J Med 1983;13:147-150.
7 Tallett ER: Stability of glyceryl trinitrate tablets. Br Med J 1982;284:1403.

8 Maclean FK, Hunt D, Marty JJ, Sloman JG, Shaw J: An assessment of the clinical use of glyceryl trinitrate in a hospital out-patient population. Aust NZ J Med 1980;10:12-14.

9 Van den Burg MJ, Cooper WD, Kimber GR: Does the lack of patient knowledge about glyceryl trinitrate limit the usefulness of the drug? $\mathrm{Br}$ J Clin Pharmacol 1985;20:246P-247P.

10 Chevigne M, Renier J, Rigo P, Denjoulin JC, Collignon P, Kulbertus HE: Efficacy of nitroglycerin in spray form. Rev Med Intern 1980;1: 265-272.

11 Kattus AA, Alvaro AB, Zohman LR, Coulson $\mathrm{AH}$ : Comparison of placebo, nitroglycerin and isosorbide dinitrate for effectiveness of relief of angina and duration of action. Chest 1979;1: 17-23.
12 Kimichi A, Lee G, Amsterdam E, Fujii K, Krieg P, Mason DT: Increased exercise tolerance after nitroglycerin oral spray: A new and effective therapeutic modality in angina pectoris. Circulation 1983;67:124-127.

13 Parker JO, Van Koughnett KA, Farrell B: Nitroglycerin lingual spray: Clinical efficacy and dose response relationship. Am J Cardiol 1986; 57:1-5.

14 Lee G, Low R, Price J, Nguyen T, Mason DT: Efficacy of Nitrolingual ${ }^{\circledR}$-Spray. Clin Res 1980; 28:191.

15 Jones B, Kenward MG: Design and Analysis of Cross-Over Trials. London, Chapman \& Hall, 1989. 
Copyright: S. Karger AG, Basel 2000. Reproduced with the permission of S. Karger AG, Basel. Further reproduction or distribution (electronic or otherwise) is prohibited without permission from the copyright holder. 